\title{
Uso de fluoruro diamino de plata para tratamiento de lesiones de caries activa.
}

\section{Use of silver diamine fluoride for treatment of active caries injuries.}

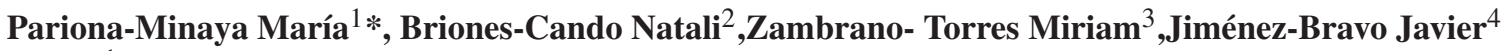 \\ ${ }^{1}$ Docente Especialista en Odontopediatría. Universidad Católica de Cuenca. Cuenca, Ecuador. \\ ${ }^{2}$ Rotante de Investigación en la Universidad Católica de Cuenca. Estudiante de pregrado de la Universidad Católica de \\ Cuenca. Cuenca, Ecuador. \\ ${ }^{3}$ Rotante de Investigación en la Universidad Católica de Cuenca, Ecuador. Odontóloga de práctica privada. \\ ${ }^{4}$ Estudiante de pregrado de la Universidad Católica de Cuenca. Cuenca, Ecuador. \\ *mparionam@ucacue.edu.ec
}

\begin{abstract}
Resumen
El Fluoruro Diamino de Plata (FDP) es un agente cariostático; el cual es idóneo en el control y detención de caries. Utilizado como un tratamiento no invasivo en odontología. Es de bajo costo y fácil manipulación, eficaz en pacientes poco o nada colaboradores y/o pacientes que no logran un fácil acceso a la consulta dental. El objetivo es reportar el uso de FDP como alternativa de tratamiento para detener la progresión de lesiones de caries activas. En el presente aporte se describe dos casos clínicos de niños de uno a cinco años de edad, que participaron en el proyecto social dirigido por la carrera de la Universidad Católica de Cuenca. Se diagnosticaron las lesiones de caries activas utilizando ICDAS y evaluando clínicamente los tejidos blandos. Clínicamente se evaluó el resultado del uso de FDP sobre piezas dentales con lesiones de caries, encontrando su detención.
\end{abstract}

Palabras clave: Caries dental; cariostático; fluoruro diamino de plata; dentición primaria; odontopediatría.

\begin{abstract}
Diamino Silver Fluoride (PDF) is a cariostatic agent; which is ideal in the control and arrest caries. Used as a non-invasive treatment in dentistry. It is low cost and easy to handle, effective in patients non-collaborators and / for patients who do not have an appointment at the dental office due to lack of financial resource. The objective is to report the use of PDF as an alternative treatment to stop the progression of active caries lesions. In this contribution, two clinical cases of children from one to five years old, who participated in the social project directed by the Universidad Católica de Cuenca, are described. Clinically, the result of the use of FDP on dental pieces with caries lesions was evaluated, finding their arrested.
\end{abstract}

Key words: Dental caries; cariostatic; silver diamine fluoride; primary dentition; pediatric dentistry.

\section{INTRODUCCIÓN}

El tratamiento restaurador convencional de las lesiones de caries conlleva un manejo invasivo ya que se debe realizar una preparación y restauración con compromiso dentario significante. Sin embargo, el mismo requiere por parte del profesional ciertas habilidades clínicas, materiales costosos y sin dejar de lado un factor importarte por parte del paciente que es su cooperación. ${ }^{1}$ En la consulta pediátrica se ha visto diferentes problemáticas entorno a niños de corta edad, necesidades especiales e incluso niños de niveles socioeconómicos bajos mostrándose obstaculizada este tipo de tratamiento tradicional en lesiones de caries extensas por lo que conducen a la progresión de la enfermedad y la pérdida de estructura dentaria incluso exponiéndose a lesiones de caries dental no tratada. Estas secuelas a largo plazo causan traumas psicológicos, problemas fonéticos, problemas en el crecimiento óseo y problemas en erupción de los dientes permanentes. ${ }^{1,2}$ Por lo que en 1891 se reportó el inicio de uso de FDP por Stebbins, realizó una mezcla de ácido nítrico y amalgama y obtiene el $61 \%$ de éxito en la desactivación de las lesiones de caries. ${ }^{3}$ En 1989 Garbelini comprueba su efecto cariogénico y cariostático aplicado en esmalte. ${ }^{4}$ Llodra en el 2005 realiza un estudio prospectivo con 2.5 a 3 años de seguimiento, observando que el $70 \%$ de las superficies tratadas con FDP, se detienen. ${ }^{5}$ En el 2016 la FDA aprobó el uso de Fluoruro Diamino de Plata(FDP) por no ser invasivo, efectivo, seguro y de fácil manipulación. Frecuentemente usado en Brasil, Hong Kong, China y Japón. 6,7

El FDP se encuentra como un fármaco de uso tópico; siendo un agente incoloro con un $\mathrm{pH}$ alcalino entre 8-10.4; presenta 
una composición de $28.8 \%$ de plata y $5.9 \%$ de fluoruro, un producto de bajo costo en las casas comerciales. El inconveniente del producto es la tinción de color negro que deja en el esmalte y dentina comprometiendo así la estética del paciente y siendo un factor para el rechazo por parte de los padres. Las casas comerciales mejoraron el producto para contrarrestar esta particularidad. Riva Star incorporó Yodo, reduciendo significativamente las manchas oscuras generadas en los órganos dentarios. ${ }^{1,8}$ Dentro de los mecanismos de acción del FDP es la formación de iones de plata y fluoruro, estos penetran 25 micras en el esmalte y 50-200 micras en la dentina. ${ }^{9}$ Las partículas de plata se ubican a lo largo de los túbulos dentinarios ayudando la formación de fluorapatita, fosfato y calcio que se forma en la lesión de caries haciendo de la dentina más dura y al diente más resistente. ${ }^{18}$ Las partículas de plata tiene acción antimicrobiana evitando la proliferación bacteriana desnaturalizando las enzimas que descompone la dentina(Matriz metaloproteinasa) evitando la degradación del colágeno orgánico que se encuentra en la dentina e inhibiendo la formación del biofilm. ${ }^{5,9,16,18}$

La formación de $\mathrm{Ag}(\mathrm{NH} 3) 2 \mathrm{~F}$ controla el proceso de remineralización adicionando el fluoruro en el interior de las capas del esmalte; en el lapso de 24 horas. ${ }^{7,17,18}$ Se ha demostrado que la tinción negruzca en la lesión de caries se produce por la reducción química del fosfato de plata al estar en contacto con el medio bucal. ${ }^{4}$

No se han reportado efectos adversos sistémicos ni muertes, se sugiere seguir las recomendaciones del fabricante. Una gota de FDP por cada $10 \mathrm{~kg}$ de peso en tratamientos semanales. El tiempo de aplicación oscila de uno a tres minutos, suave secado de las piezas dentales y no ingerir alimentos ni bebidas en un lapso de treinta minutos a una hora. ${ }^{4,9}$

La presentación de esta serie de casos clínicos tuvo como objetivo aplicar FDP en lesiones de caries activa en niños de uno a cinco años de edad con poca accesibilidad a servicios odontológicos.

\section{ESTADO DEL ARTE}

En la actualidad existen varias terapéuticas que se utilizan para tratar la caries, todas estas se basan en desgastar y quitar el tejido dañado para ser remplazado por un material restaurador. Desde 1970 se ha utilizado el FDP, pero en las últimas dos décadas estas se dejó de usar de manera significativa por la creciente importancia debido a la estética que asume la gente que debe tener la pieza dental. Actualmente existe un movimiento conservador que utiliza diferentes tipos de flúor en los que se han incluido nuevamente el FDP. Para crear un protocolo y comprobar su eficacia se utiliza este producto en una clínica externa de la carrera de Odontología de la Universidad Católica de Cuenca. En octubre 2019 a febrero 2020 especialistas en odontopediatría y estudiantes de pregrado evaluaron un promedio de setenta niños de uno a cinco años de edad de la Unidad Educativa Alfonso Carrión, Cuenca-Ecuador. Se realizó la anamnesis, odontograma y observamos que el $60 \%$ de los niños evaluados presentaban lesiones de caries activas e inactivas con sensibilidad. En la figura 1 se observa el paciente A con lesiones de caries activas y perdida de estructura en vestíbulo-cervical de las piezas 52,51, 61 y 62. Para la colocación de diamino de plata seleccionamos los dientes sin alteraciones pulpares ni periodontales y con lesiones de caries activas. Dentro de los criterios para la selección de la pieza tuvimos en cuenta que en tejidos blandos no tuviésemos edema y eritema. No hubiese compromiso pulpar con ausencia en el fondo de surco absceso y fistulas.

Las piezas dentales debían presentar lesiones de caries con dentina afectada y un techo pulpar firme sin exposición pulpar.

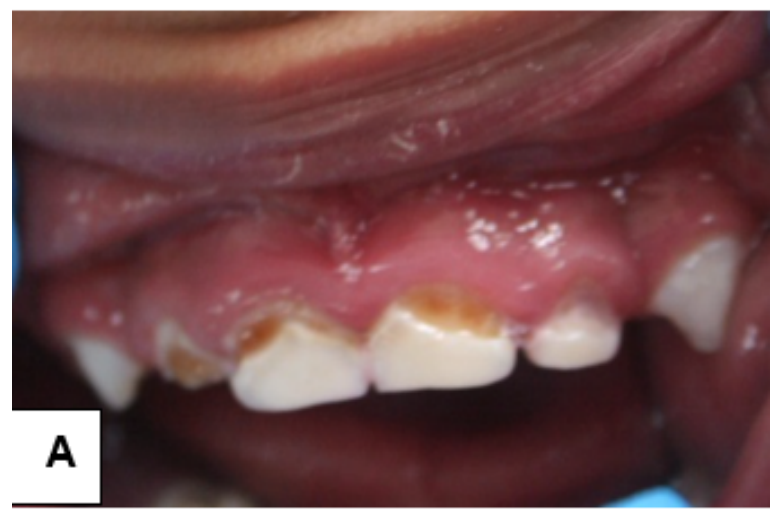

Fig. 1.

Primero se redactó el consentimiento informado para enviarlo a los padres de familia, con mayor énfasis en la particularidad de la tinción negra que produciría el FDP en las piezas dentarias tratadas. Se adjuntaron al consentimiento fotografías del posible resultado final y se mencionó que la consecuencia a la aplicación seria la tinción obscura de las caries. El asentimiento del niño también fue requerido para iniciar el tratamiento. Sin embargo, en niños menores de tres años se utilizó restricción física activa.

En la primera sesión, se procedió a realizar la técnica de cepillado con pasta de 1100 ppm de flúor con el objetivo de eliminar la placa dental y dejar una superficie limpia para la aplicación del agente cariostático como se observa en el paciente B. (Figura 2) 


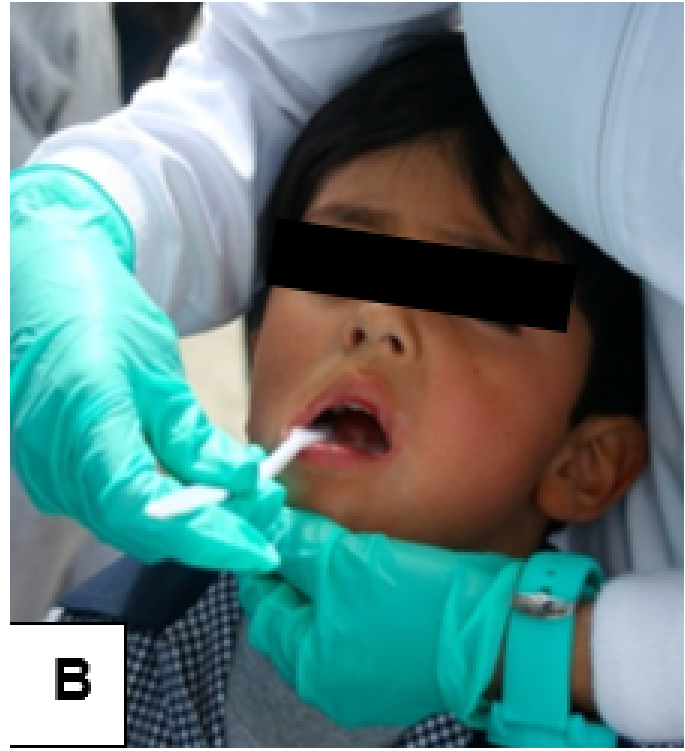

Fig. 2.

Se adecuó el espacio para el desarrollo del tratamiento mediante la colocación de plástico en las sillas y mesas escolares. El instrumental utilizado fue vaselina, royos de algodón, gasas estériles, kit de diagnóstico, vaso dappen, pincel, microbrush, servilletas y el fluoruro diamino de plata al 30\%(Cariestop) frasco $5 \mathrm{ml}$ de la casa comercial Biodinámica-Brasil. (Figura 3) Se le colocó al paciente un campo de papel como protección a posibles salpicaduras.

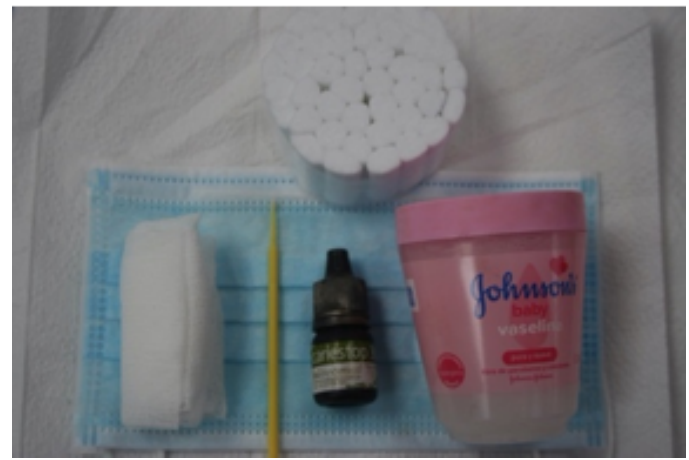

Fig. 3 .

Posteriormente se realizó el aislamiento relativo de los tejidos blandos con royos de algodón en vestibular para dientes anteriores y en lingual-vestibular para dientes posteriores. Se colocó vaselina en labios, mucosa, encías, fondo de surco y punta de la lengua con el objetivo de evitar pigmentaciones e irritaciones no deseadas como se observa en la figura 4 del paciente B. Se colocó en la superficie cóncava del vaso dappen una gota del producto por cada dos dientes y dos gotas por cuatro lesiones de caries.

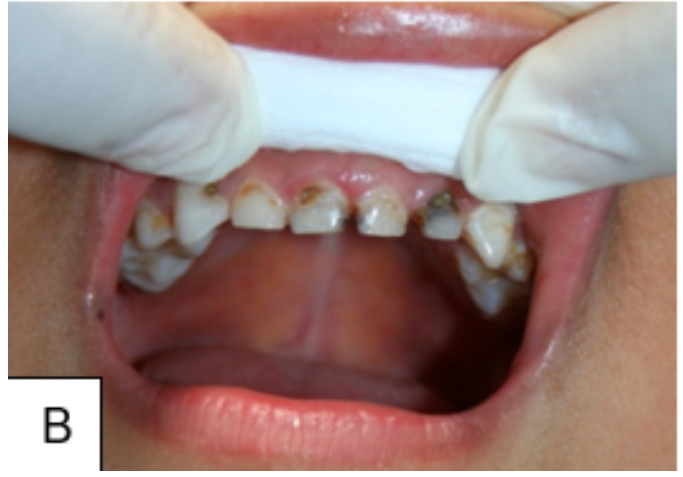

Fig. 4.

Se continuó con el secado de las piezas dentales con gasas estériles, se sumergió el microbrush en la gota de FDP y se aplicó directamente en las lesiones de caries dos veces como se observa en la figura 5 de los pacientes A y B. (Figura 5)

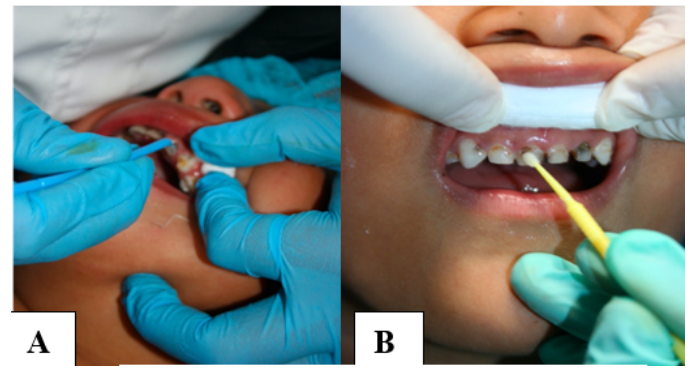

Fig. 5.

Se dejó el producto durante un minuto, pues la literatura nos refleja que es el tiempo necesario para que ejerza su mecanismo de acción. Luego se eliminó el exceso de igual manera con una torunda de algodón, se procedió a lavar con agua y por último se retiró las torundas utilizadas para el aislamiento relativo. Se humedeció los labios para evitar lesiones futuras y se dio por concluido el procedimiento. El personal dio continuidad con los protocolos de bioseguridad, retirándose los guantes y desechándolos junto con los instrumentales antes mencionados en los lugares designados para desechos biológicos. En la segunda visita fue necesario comprobar que el diente no haya tenido complicación pulpar, verificado el diagnóstico, se procedió a una segunda aplicación. Reportamos que el paciente A refirió una significativa disminución a la sensibilidad y mejoría en la masticación durante la alimentación. Desde la tercera visita se realizó los controles, en el cual se evidenciaba la evolución en la detención de la lesión de caries comprobando así la eficacia del FDP. (Figura 6) Se desactivó el $100 \%$ de las lesiones de caries activas que fueron diagnosticadas en los setenta niños que participaron en el programa. 


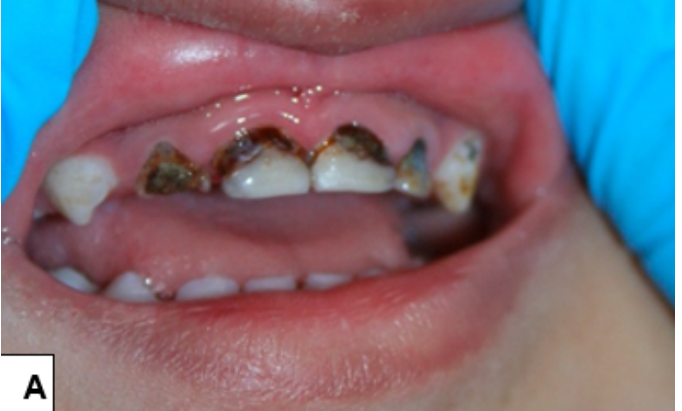

Fig. 6.

\section{DISCUSIÓN}

Según la revisión de la literatura, Ecuador tiene escasos reportes sobre el FDP utilizado como cariostático en detención de lesiones de caries activa; se presume que la razón es la difícil obtención del producto; pero a nivel internacional estudios han demostrado su eficacia para desactivar lesiones de caries en dientes deciduos como menciona Contreras V. 2017 la concentración de FDP al 30\% y $38 \%$ cumple con el efecto de manejo preventivo para la detención de las lesiones cariosas. ${ }^{18,19}$ En personas con diferentes necesidades pudiendo ofrecer al paciente, tutor y al especialista una mejor experiencia en la consulta.

Según Horst. $2017^{9}$ para la aplicación de FDP el profesional debe tener en cuenta otros factores como la frecuencia de consumo de azúcar, uso de cepillado dental y la exposición de fluoruros de forma sistémica y tópica. El incumplimiento de estos parámetros lleva a la reactivación de las lesiones desactivadas. La evidencia nos muestra que la aplicación de esta sustancia acompañada de un seguimiento correcto por parte del odontólogo garantiza la permanencia del diente deciduo en boca hasta que este se exfolie.

Chu Ch. $2002^{10}$ realizó un estudio comparativo entre fluoruro de diamino de plata con una concentración de 44.800 ppm F y barniz de fluoruro de sodio con 22.600 ppm F en niños de 3-5 años preescolares de la población China en un ensayo clínico prospectivo controlado la cual se comparó su eficacia cada tres meses. El cual se vio con mayores resultados a pesar de no ser significativos al FDP como un cariostático en dientes primarios anteriores.

Jiang M. $2020^{11}$ mediante su revisión sistemática evalúa sobre la influencia de la aplicación en la fuerza de unión de la dentina hacia el FDP, adhesivos y a los cementos de Ionómero de vidrio no se obtuvieron diferencias considerables a pesar de que las concentraciones, protocolos y cantidades son acorde al producto.

Duangthip D. $2018^{13}$ descubrió mayor eficacia en las aplicaciones anuales de FDP muy contrario a las tres aplicaciones semanales de barniz de $\mathrm{NaF}$ para la detención de lesiones de caries activa en dentición primaria.12 Vinod D. 2020 evaluó el potencial de remineralización de FDP, fosfato de caseína sacarosa, fosfato de calcio amorfo de péptido de caseína fosfato mediante el análisis de DIAGNOdent; comprobando valores de remineralización altos en los grupos tratados con FDP siendo esta no invasiva y a fin a niño de edades tempranas.

Gao S. $2016^{14}$ menciona el uso de FDP al $38 \%$ como un tratamiento eficaz para la detención de caries en niño sin embargo el sugiere nuevos estudios para generalizar pautas basadas en evidencia para el uso en el área pediatrica. Mientras que Zhi QH. $2012^{15}$ indica la efectividad de FDP y un Ionómero de vidrio fluido de alta liberación de fluoruro frente a la detención de caries viendo que ofrecen los mismos resultados, pero con mayor resultado estético muestra el Ionómero de vidrio fluido.

\section{CONCLUSIONES}

El FDP es una sustancia que desactiva las lesiones de caries, de fácil manipulación y con respaldo científico. Uno de los efectos adversos es la pigmentación de las lesiones de las caries en los dientes. Recomendamos el uso de FDP en pacientes poco o nada colaboradores debido a que el protocolo de aplicación es simple y práctico. Ayuda a la modificación de conducta y una vez desactivada la lesión, nos permite planificar una intervención más estética y con menos efecto traumático.

Sugerimos el uso del FDP como cariostático para lesiones de caries activas, en estos tiempos de pandemia por el virus SARS-COV2. Tiene un enfoque preventivo, evita el avance de lesiones de caries iniciales que puedan generar dolor y compromiso pulpar si no reciben tratamiento. Otro beneficio que observamos es que no requiere el uso de pieza de mano disminuyendo la producción de aerosoles evitando así la contaminación cruzada en la consulta. 
CONFLICTO DE INTERESES: Los autores 13 Vinod D, Gopalakrishnan A, Subramani SM, Balachandran

manifiestan no tener conflicto de interés.

Mención especial al Departamento de Vinculación con la sociedad de la Carrera de Odontología de la Universidad Católica de Cuenca y a los alumnos que participaron en este proyecto.

\section{Referencias Bibliográficas}

1 Galui S., Pal S., Pabale SL., Saha S., Sarkar S. Stretching New Boundaries of Caries Prevention with Silver Diamine Fluoride: A Review of Literature. Int J Pedod Rehabil. 2018; 3:1-4.

2 Seifo N., Cassie H., Radford J., Innes N. Silver diamine fluoride for managing carious lesions: an umbrella review. 2019; 19:145.

3 Chibinski A., Wambier L., Feltrin J., Luguercio A., Wambier D., Reis D. Silver Diamine Fluoride Has Efficacy in controlling Caries Progression in Primary Teeth: A Systematic Review and Meta-Analysis. Karger AG, Basel. 2017 Oct, 51: 527-541.

4 Elias M., Fluoruro diaminico de plata: Técnica de pincel y Vaselina. Gaceta Odontológica. 2018 May; 88 (2): 116-125.

5 Orellana J., Morales V., González M., Fluoruro diamino de plata: Su utilidad en la odontología pediátrica. ResearchGate. 2019 Abril - Junio; 6(2): 57 - 60.

6 Horst JA, Heima M. Prevention of Dental Caries by Silver Diamine Fluoride. Compend Contin Educ Dent. 2019 Mar;40(3):158-163.

7 Orellana-Centeno JE., Morales-Castillo V, González-Osorio M. Fluoruro diamino de plata: Su utilidad en la odontología pediátrica. Avan C Salud Med. 2019; 7 (2):57-60.

8 Mendoza- Cavero MC., Ortiz-Velasquez MA., MatounFarah M. Fluoruro Diamino de Plata (FDP) al $38 \%$. Su uso en pacientes odontopediátricos y con necesidad especiales. Protocolo de aplicación. Rev Orto y Odont. 2020

9 Horst JA, Ellenikiotis H, Milgrom PL. Protocolo UCSF para el arresto de caries usando fluoruro de diamina de plata: justificación, indicaciones y consentimiento. J Calif Dent Assoc. 2016 enero; 44 (1): 16-28.

$10 \mathrm{Chu} \mathrm{CH}$, Lo EC, Lin HC. Effectiveness of silver diamine fluoride and sodium fluoride varnish in arresting dentin caries in Chinese pre-school children. J Dent Res. 2002 Nov;81(11):767-70.

11 Jiang, M., Mei, ML, Wong, MCM y col. Efecto de la aplicación de la solución de fluoruro de diamina de plata sobre la fuerza de unión de la dentina a los adhesivos y a los cementos de Ionómero de vidrio: una revisión sistemática. BMC Oral Health.2020; 40.

12 Duangthip D, Wong MCM, Chu CH, Lo ECM. Caries arrest by topical fluorides in preschool children: 30-month results. J Dent. 2018 Mar; 70:74-79.
M, Manoharan V, Joy A. A Comparative Evaluation of Remineralizing Potential of Three Commercially Available Remineralizing Agents: An In Vitro Study. Int J Clin Pediatr Dent. 2020 Jan-Feb;13(1):61-65.

14 Gao, S. S., et al. Çlinical trials of silver diamine fluoride in arresting caries among children: a systematic review."JDR Clinical \& Translational Research. 2016;1(3): 201-210.

15 Zhi QH., Lo EC., Lin HC. Randomized clinical trial on effectiveness of silver diamine fluoride and glass ionomer in arresting dentine caries in preschool children. J Dent. 2012 Nov;40(11):962-7.

16 Burgess JO., Vaghela P. M. Silver diamine fluoride: a successful anticarious solution with limits. Advances in dental research. 2018; 29(1):131-134.

17 Milgrom P, Horst J, Ludwig S. Topical silver diamine fluoride for dental caries arrest in preschool children: A randomized controlled trial and microbiological analysis of caries associated microbes and resistance gene expression. J Dent. 2018; 68:72-78.

18 Zhao IS., Gao SS., Hiraishi N. Mechanisms of silver diamine fluoride on arresting caries: a literature review. International dental journal. 2018;68(2):67-76.

19 Contreras V., Toro M., Boneta AR., Burgos E. Effectiveness of silver diamine fluoride in caries prevention and arrest: a systematic literature review. General dentistry. 2017;65(3): 22.

Recibido: 21 de julio dell 2020

Aceptado: 20 de agosto del 2020 
